\title{
Use of PCR-based Markers for Differentiating Elite Broccoli Inbreds
}

\author{
Anna L. Hale ${ }^{1}$ and Mark W. Farnham ${ }^{1}$ \\ USDA/ARS U.S. Vegetable Laboratory, Charleston, SC 29414 \\ Monica A. Menz ${ }^{2}$ \\ Institute for Plant Genomics and Biotechnology, Department of Soil and Crop Science, Texas A\&M \\ University, College Station, TX 77843
}

\begin{abstract}
ADDITIONAL INDEX words. Brassica oleracea, SSR, AFLP, SRAP
Abstract. Breeders of cole crops (Brassica oleracea L.) have an interest in utilizing current and emerging PCR-based marker systems to differentiate elite germplasm. However, until efficiency and cost-effectiveness are determined, most breeders are hesitant to change methods. In this study, our goal was to compare simple sequence repeat (SSR), amplified fragment-length polymorphism (AFLP), and sequence-related amplified polymorphism (SRAP) marker systems for their effectiveness in differentiating a diverse population of 24 elite broccoli (B. oleracea Italica Group) inbreds. Published SSR primer sequences for Brassica $\mathrm{L}$. species were used along with AFLP and SRAP primer combinations. Several SSR primers failed to amplify DNA in the broccoli population, but all AFLP and SRAP primer combinations produced multiple bands. Twenty-nine percent of the SSR primers were monomorphic, while most of the remaining primers detected only one or two differences among inbreds. AFLP and SRAP methods produced multiple differences per primer in almost every case. Phenetic analysis revealed that the type of marker affected the classification of the genotypes. All three marker systems were able to successfully differentiate between the 24 elite inbreds, however, AFLPs and SRAPs were more efficient, making them better alternatives than SSRs over other established methods for fingerprinting $B$. oleracea inbreds.
\end{abstract}

Commercial and public broccoli breeders have an ongoing interest in utilizing current and emerging PCR-based marker systems to differentiate elite germplasm in their vegetable improvement programs. Any potential new marker systems must be more efficient and cost-effective alternatives to older techniques already in use by the respective programs. Until efficiency and cost-effectiveness are determined, most breeders are hesitant to change their methods.

The first DNA-based marker system widely used by Brassica breeders was restriction fragment length polymorphisms (RFLPs). These markers have been used to study genetic similarity, taxonomy, and genome evolution (dos Santos et al., 1994; Neinhuis et al., 1993; Song et al., 1988). While RFLPs have proven to be useful co-dominant markers, they have two major limitations. First, RFLPs are time consuming, and results are limited only to the DNA sequence used as a probe, and thus, a small area of the genome. Additionally, most effective RFLP methods require the use of radioactivity.

Random amplified polymorphic DNA (RAPD) markers were the first widely used alternative to RFLPs (Williams et al., 1990). Using RAPDs, large numbers of samples could be analyzed economically and quickly. The method required small quantities of DNA, and most of the genome could be sampled with a potentially unlimited number of markers. RAPDs have been used for cultivar identification (Hu and Quiros, 1991; Lee et al., 1996), studies on genetic diversity among populations (Farnham, 1996; Kaminski et al., 2003; Lanner-Herrera et al., 1996), gene bank management (Divaret et al., 1999; Kresovich et al., 1992; Phippen

Received for publication 1 Dec. 2005. Accepted for publication 25 Feb. 2006. The contents of this publication do not necessarily reflect the views or policies of the USDA, nor does the mention of trade names, commercial products, or organizations imply endorsement by the U.S. Government.

${ }^{1}$ Research Geneticist.

${ }^{2}$ Assistant Professor. et al., 1997) and identification and mapping of markers (Camargo et al., 1997; Giovannelli et al., 2002). Based on the number of past and recent publications on RAPDs and the frequent use of them by private companies, they may still be the most common PCR-based marker system used in B. oleracea breeding programs. Despite the advantages RAPDs have over RFLPs, they tend to be dominant markers, have low multiplex ratios (meaning they produce few markers per primer) and poor consistency between different breeding programs. These disadvantages have put them out of favor by many geneticists.

Following wide usage of RAPDs in Brassica breeding, new PCR-based techniques have been developed. Each of these techniques has its advantages and disadvantages. AFLPs have a high multiplex ratio, are less sensitive to the template DNA concentrations, and are highly reproducible (Becker et al., 1995; Breyne et al., 1999; Vos et al., 1995). The primary disadvantages of this method include the multiple steps involved in the process, the time required for optimization, potentially uneven marker distribution (Haanstra et al., 1999), and the difficulty in isolating specific bands for sequencing. AFLPs have been used in B. oleracea to identify quantitative trait loci and for generating linkage maps (Holme et al., 2004; Sebastian et al., 2000). Previous work with a multitude of species has shown that SSRs, while lacking the high multiplex ratio seen in AFLPs and requiring prior sequence knowledge to design primers, are co-dominant and exhibit a much higher degree of polymorphism than do any other markers (Bowers et al., 1996). While SSRs have not been used extensively on $B$. oleracea, they have been utilized in related Brassica species for genome identification and to study genome evolution (Lowe et al., 2002; Plieske and Struss, 2001; Saal et al., 2001). A relatively new PCR-based technique, SRAPs, is gaining popularity due to its simplicity, reliability, a higher frequency of co-dominant markers, moderate through-put ratio, and their tendency to map in close proximity to expressed regions of the genome ( $\mathrm{Li}$ and Quiros, 2001). Despite their advantages and successful use in 
related Brassica species, AFLPs, SRAPs, and SSRs have not been as widely utilized as RAPDs in B. oleracea research.

The objective of the current study was to compare the informativeness of SSR, AFLP, and SRAP markers for their ability to discriminate between a set of broccoli inbreds and to determine the feasibility of using these markers in place of older techniques, such as RAPDs, that are typically used in B. oleracea breeding programs.

\section{Materials and Methods}

Plant materials and DNA extraction. A population of 24 elite broccoli inbreds was selected to represent a diverse phenotypic and genotypic array of parental broccoli material that might typically occur in a breeding program aimed at developing elite cultivars. Fourteen of the lines are doubled haploids developed using standard techniques for anther culture (Farnham, 1998). These are designated USVL012, USVL089, and USVL013 derived from 'Everest'; USVL047, USVL048, and USVL073 derived from 'Marathon'; USVL105 from 'Arcadia'; USVL032 from 'Green Valiant'; USVL070 from 'Futura'; USVL066 from 'Viking'; USVL039 from 'High Sierra'; USVL107 from 'Patriot'; USVL106 from 'Major'; and USVL093 from 'Sultan'. The population also included 10 inbreds developed through conventional pedigree selection. These include USVL110, USVL111, USVL059, USVL060, and USVL064 (derived from 'Green Comet', 'Paragon', 'Packman', 'Southern Comet', and 'Symphony', respectively) developed at the U.S. Vegetable Laboratory (USVL) in Charleston, S.C.; USVL112 and USVL113 selected at the USVL from heterozygous lines obtained from M. Dickson (Cornell Univ.); S240-5-20 and S440 obtained from J. Myers (Oregon State Univ.), and USVL109 developed at the USVLfrom a heterozygous line from Syngenta (Boise, Idaho).

Genomic DNA was isolated from leaves using DNeasy plant mini-kits (Qiagen, Valencia, Calif.). DNA was quantified using a TKO 100 fluorimeter (Hoefer Scientific Instruments, San Francisco) in conjunction with a Hoechst dye-based protocol.

SSR ANALYSIS. The test population of 24 broccoli inbreds was screened with 44 previously identified SSR primers. Fifteen primers were identified by Suwabe et al. (2002), seven by Lowe et al. (2002), seven by Kresovich et al. (1995), and 15 by Szewc-McFadden et al. (1996). Of the 44 SSRs used in this study, 15 were identified in B. rapa L., 24 in B. napus L., three in B. nigra L., and two in $B$. oleracea (Table 1). PCR was performed in $10-\mu \mathrm{L}$ reactions containing $1 \mathrm{X}$ PCR buffer (Promega, Madison, Wis.), $2.5 \mathrm{~mm} \mathrm{MgCl}_{2}, 100 \mu \mathrm{M}$ dNTPs (Sigma, St. Louis), $0.25 \mu \mathrm{M}$ of each primer pair (Sigma Genosys, Houston), 0.2 U Taq DNA polymerase (Promega), and 25 ng of DNA. The PCR conditions were identical to that of Saal et al. (2001), and annealing temperatures ranged from 49 to $68{ }^{\circ} \mathrm{C}$ (Table 1 ). The PCR products were separated on a $7 \%$ native polyacrylamide gel with ethidium bromide in the running buffer and visualized on a ultraviolet transilluminator (Shi et al., 2001).

SRAP ANALYSIS. Twenty-four SRAP primer combinations were evaluated on the test population using seven forward primers and eight reverse primers (Table 2). The seven forward primers and three of the reverse primers were previously described by $\mathrm{Li}$ and Quiros (2001), and the remaining reverse primers were developed according to the rules for primer design as outlined in the same paper. For visualization purposes, the forward primers were all labeled with one of three WellRED dyes (D2, D3, or D4; Proligo, Boulder, Colo.), and to ensure proper dye balance, the amount of
Table 1. SSR primers, the source of the sequence, and the respective annealing temperatures (TA) used to analyze 24 elite broccoli inbreds.

\begin{tabular}{|c|c|c|c|}
\hline Primer & Source & Species & $\mathrm{TA}$ \\
\hline$\overline{\text { BRMS001 }}$ & Suwabe et al. (2002) & Brassica rapa & 54 \\
\hline BRMS005 & Suwabe et al. (2002) & B. rapa & 52 \\
\hline BRMS007 & Suwabe et al. (2002) & B. rapa & 51 \\
\hline BRMS008 & Suwabe et al. (2002) & B. rapa & 53 \\
\hline BRMS017 & Suwabe et al. (2002) & B. rapa & 55 \\
\hline BRMS019 & Suwabe et al. (2002) & B. rapa & 49 \\
\hline BRMS020 & Suwabe et al. (2002) & B. rapa & 48 \\
\hline BRMS025 & Suwabe et al. (2002) & B. rapa & 65 \\
\hline BRMS002 & Suwabe et al. (2002) & B. rapa & 59 \\
\hline BRMS003 & Suwabe et al. (2002) & B. rapa & 51 \\
\hline BRMS006 & Suwabe et al. (2002) & B. rapa & 56 \\
\hline BRMS014 & Suwabe et al. (2002) & B. rapa & 57 \\
\hline BRMS015 & Suwabe et al. (2002) & B. rapa & 60 \\
\hline BRMS016 & Suwabe et al. (2002) & B. rapa & 64 \\
\hline 0109-A03 & Lowe et al. (2002) & B. oleracea & 50 \\
\hline 0109-A06 & Lowe et al. (2002) & B. oleracea & 59 \\
\hline NA10-B10 & Lowe et al. (2002) & B. napus & 59 \\
\hline NA10-F06 & Lowe et al. (2002) & B. napus & 63 \\
\hline $\mathrm{NI} 2-\mathrm{B} 02$ & Lowe et al. (2002) & B. nigra & 58 \\
\hline $\mathrm{NI} 2-\mathrm{C} 12$ & Lowe et al. (2002) & B. nigra & 58 \\
\hline NI-F02 & Lowe et al. (2002) & B. nigra & 56 \\
\hline BRMS044 & Suwabe et al. (2002) & B. rapa & 60 \\
\hline BN72A & Szewc-McFadden et al. (1996) & B. napus & 61 \\
\hline BN38A & Szewc-McFadden et al. (1996) & B. napus & 65 \\
\hline BN12A & Szewc-McFadden et al. (1996) & B. napus & 62 \\
\hline BN6A2 & Szewc-McFadden et al. (1996) & B. napus & 65 \\
\hline BN6A3 & Szewc-McFadden et al. (1996) & B. napus & 62 \\
\hline BN9A & Szewc-McFadden et al. (1996) & B. napus & 55 \\
\hline BN16A & Szewc-McFadden et al. (1996) & B. napus & 66 \\
\hline BN18A1 & Szewc-McFadden et al. (1996) & B. napus & 65 \\
\hline BN20A & Szewc-McFadden et al. (1996) & B. napus & 56 \\
\hline $\mathrm{BN} 25 \mathrm{~A}$ & Szewc-McFadden et al. (1996) & B. napus & 60 \\
\hline BN19A & Szewc-McFadden et al. (1996) & B. napus & 63 \\
\hline $\mathrm{BN} 25 \mathrm{C} 2$ & Szewc-McFadden et al. (1996) & B. napus & 62 \\
\hline BN26A & Szewc-McFadden et al. (1996) & B. napus & 61 \\
\hline $\mathrm{BN} 72 \mathrm{~B} 2$ & Szewc-McFadden et al. (1996) & B. napus & 68 \\
\hline BN40C1 & Szewc-McFadden et al. (1996) & B. napus & 59 \\
\hline BN50F & Szewc-McFadden et al. (1996) & B. napus & 52 \\
\hline BN59A1 & Szewc-McFadden et al. (1996) & B. napus & 64 \\
\hline BN68/1 & Szewc-McFadden et al. (1996) & B. napus & 64 \\
\hline BN75A & Szewc-McFadden et al. (1996) & B. napus & 60 \\
\hline $\mathrm{BN} 80 / 3$ & Szewc-McFadden et al. (1996) & B. napus & 63 \\
\hline BN83B1 & Szewc-McFadden et al. (1996) & B. napus & 59 \\
\hline BN92A1 & Szewc-McFadden et al. (1996) & B. napus & 60 \\
\hline
\end{tabular}

dye-labeled primer added to the PCR reaction varied depending on which dye was used. Each $10-\mu \mathrm{L}$ PCR reaction contained $1 \mathrm{X}$ PCR buffer (Promega), $2.5 \mathrm{~mm} \mathrm{MgCl}, 200 \mu \mathrm{M}$ dNTPs (Sigma), $30 \mathrm{ng}$ of reverse primer (IDT, Coraville, Iowa), 0.4 U Taq DNA polymerase (Promega) and $25 \mathrm{ng}$ of DNA. In addition, WellRED dye labeled forward primers were added in the following amounts: $15 \mathrm{ng}$ for a D4 primer; $30 \mathrm{ng}$ for a D3 primer; or $75 \mathrm{ng}$ for a D2 primer. PCR conditions were identical to Li and Quiros (2001), except 40, as opposed to 35, cycles were performed. The PCR products were prepared for running on a CEQ8800 capillary sequencer (Beckman Coulter, Fullerton, Calif.) by pooling 0.3 $\mu \mathrm{L}$ of the $\mathrm{D} 4$ reaction, $0.3 \mu \mathrm{L}$ of the $\mathrm{D} 3$ reaction, $0.5 \mu \mathrm{L}$ of the D2 reaction, $0.28 \mu \mathrm{L}$ of D1 size standard, and $30 \mu \mathrm{L}$ of de-ionized formamide in a 96-well PCR plate. Samples were run on the 
Table 2. Primer sequences for SRAP reactions.

\begin{tabular}{lll}
\hline Primer & Direction & \multicolumn{1}{c}{ Sequence } \\
\hline ME1 & Forward & TgAgTCCAAACCggATA \\
ME2 & Forward & TgAgTCCAAACCggAgC \\
ME3 & Forward & TgAgTCCAAACCggAAT \\
ME4 & Forward & TgAgTCCAAACCggACC \\
ME5 & Forward & TgAgTCCAAACCggAAg \\
AB2 & Forward & AgTgATTCAACCggATA \\
AB3 & Forward & AgTgATTCAACCggAgC \\
EM1 & Reverse & gACTgCgTaCgAATTAAT \\
EM5 & Reverse & gACTgCgTACgAATTAAC \\
EM6 & Reverse & gACTgCgTACgAATTgCA \\
BA1 & Reverse & gTCgAgCTgCCAATTATA \\
BA2 & Reverse & gTCgAgCTgCCAATTAAT \\
BA3 & Reverse & gTCgAgCTgCCAATTTgC \\
BA4 & Reverse & gTCgAgCTgCCAATTTTT \\
BA5 & Reverse & gTCgAgCTgCCAATTAAA \\
\hline
\end{tabular}

CEQ8800 capillary sequencer using the fragment 3 parameters and analyzed using the built-in fragment analysis software that was bundled with the system. A relative peak height threshold of $10 \%$ was used for calling the peaks. The fragments between 75 and 400 bp were scored as present (1) or absent (0).

AFLP ANALYsis. AFLP analysis was conducted according to Vos et al. (1995) with minor modifications. Genomic DNA was restricted with $5 \mathrm{U}$ of EcoRI (rare cutter) and $5 \mathrm{U}$ of $M s e \mathrm{I}$ (frequent cutter) (New England Biolabs, Beverly, Mass.) and the double stranded adapters were ligated to the fragment ends. Preselective amplification was carried out according to Klein et al. (2000) with minor modifications. $25-\mu \mathrm{L}$ reactions contained 1X PCR buffer, $1.5 \mathrm{~mm} \mathrm{MgCl}_{2}, 160 \mu \mathrm{M}$ dNTPs, $0.56 \mu \mathrm{M}$ Eco+A and Mse+C pre-amplification primers, 1 U Taq DNA polymerase, and $5 \mu \mathrm{L}$ of restricted adapter-ligated, diluted DNA. The primer sequences were previously given by Vos et al. (1995), and the PCR profile was identical to that of Klein et al. (2000), except 25, as opposed to 20 , cycles were performed. The resulting product was diluted 1:10 with water and run on a 1\% agarose gel to verify amplification.

Following preselective amplification, selective amplification was performed using primers with three selective bases on the 3 ' end (Vos et al., 1995). For visualization purposes, the EcoRI selective primer was labeled with one of three WellRED dye labels (D2, D3, or D4). Twenty-four selective amplification primer combinations were tested on the test population using three WellRED dye labeled forward primers and eight unlabeled reverse primers. The primer combinations included AGA(D4)-CCA, CGA, CGG, CGC, CGT, CCC, CCT, CCG, and CTC; ACC(D3)-CCA, CGA, CGG, CGC, CGT, CCC, CCT, CTC, and CCG; ACT(D2)- CCA, CGT, CCC, CCT, CCG, and CTC. Reactions are the same as those described above for SRAP analysis. The PCR conditions were as follows. Cycle 1 began with a 2-min 20-s denaturation step at $94{ }^{\circ} \mathrm{C}$ followed by a 30 -s annealing step at $65^{\circ} \mathrm{C}$ and a 2 -min extension at $72{ }^{\circ} \mathrm{C}$. Cycle 2 was similar to the first cycle; however, the denaurating step was for 2 min only. Cycle 3 began at $94{ }^{\circ} \mathrm{C}$ for $20 \mathrm{~s}$ followed by an annealing step at $63^{\circ} \mathrm{C}$ for $30 \mathrm{~s}$, and a 2 -min extension at $72^{\circ} \mathrm{C}$. The annealing step was reduced by $1{ }^{\circ} \mathrm{C}$ per cycle for the next seven cycles, giving a touch down phase of eight cycles. Thirty-five cycles were then performed with a 20 -s denaturing step at $94{ }^{\circ} \mathrm{C}$, a 30 -s annealing step at 56 ${ }^{\circ} \mathrm{C}$ and a 2 -min extension at $72{ }^{\circ} \mathrm{C}$. Following these 35 cycles, an additional extension step was performed at $60^{\circ} \mathrm{C}$ for $30 \mathrm{~min}$. Samples were pooled, visualized, and analyzed as described above for SRAPs.
DATA ANALYSis. AFLP and SRAP markers were scored as present (1) or absent (0), and percent polymorphism of the markers were determined. For SSRs differences in band size were scored as different allelic forms of a given locus, and the data were transformed to binary presence (1) vs. absence (0). The genetic similarity between each pair of lines was calculated according to the Dice similarity coefficient (Nei and Li, 1979). Values of genetic similarity range from 0 (no peaks in common) to 1 (identical peak patterns for all markers). Monomorphic peaks were not included in the analysis.

Four similarity matrices were generated for comparing the marker systems. These matrices were generated using the following data sets: 1) SSR data only, 2) SRAP data only, 3) AFLP data only, and 4) a concatenated dataset of SSR, AFLP, and SSR data together. Dendrograms were created from each similarity matrix by the unweighted pair group method with arithmetic average [UPGMA(Sneath and Sokal, 1973)]. Acophenetic matrix was generated for each of the dendrograms and then compared to its corresponding similarity matrix using the Mantel matrix correspondence test (Mantel, 1967). Dendrograms created using different marker systems were compared by estimating the correlation among their corresponding cophenetic matrices and similarity matrices using the same Mantel test. The cophenetic correlation generated by the Mantel test is used as a measure of goodness of fit for a cluster analysis; 1000 permutations were used in each of our Mantel tests. Genetic similarities, similarity matrices, dendrograms, and Mantel tests were all performed by the appropriate routines in NTSYS-pc version 2.0 (Exeter Software, Setauket, N.Y.). A bootstrap analysis was performed using the WinBoot software (Yap and Nelson, 1996) to estimate the confidence limits for the groupings produced by each dendrogram; 5000 bootstrap resamplings were used for each dendrogram presented.

\section{Results and Discussion}

LEVELS OF POLYMORPHISM. Each of the three marker types evaluated were able to successfully differentiate the diverse broccoli inbreds in the test population; however, the levels of polymorphism detected varied between the marker systems.

No amplification was observed for five of the 44 SSR primer pairs, and three others were either noisy or had poor amplification. The remaining 36 primer pairs produced a total of 87 apparent loci, 55 of which were polymorphic. Fifteen SSR primers $(29 \%)$ produced a monomorphic marker phenotype. A high level of monomorphic bands was also reported in a SSR study on B. napus where only one-third of the SSR primers tested were polymorphic (Sobotka et al., 2004). Of the 36 primer pairs analyzed, an average of two loci per SSR primer pair was observed and $63 \%$ of these loci were polymorphic. The SSRs were not analyzed on the Beckman CEQ8800 capillary sequencer because it was not cost efficient to label individual primers that yield so few bands per primer.

At the beginning of the study, eight SRAP primer combinations, eight AFLP primer combinations, and eight SSRs were run in duplicate to test for reproducibility. Since no problems were observed, and previous reports indicated they were highly reproducible, the methods were deemed robust and the remaining primers were run only once.

The 24 SRAP primer combinations produced a total of 312 scoreable peaks, with an average of 13 per primer combination; $255(82 \%)$ of these peaks were polymorphic. Between 4 and 24 
fragments were produced per primer combination. The number of polymorphic fragments per primer combination ranged from one to 23 , with an average of 11 .

AFLPs produced more peaks than SRAPs or SSRs with a total of 510 for the 24 primer combinations. This averaged to 21 peaks per primer combination, with a polymorphism rate of $61 \%$. Between 10 and 40 fragments were produced per primer combination. The number of polymorphic fragments per primer combination ranged from three to 26 with an average of 13.

GENETIC SIMILARITY. Genetic similarity over all marker systems among the 24 lines ranged from 0.32 to 0.91 . SSRs had genetic similarities ranging from 0.32 (between USVL070, and USVL111, derived from 'Futura' and 'Paragon', respectively) and 0.91 (between S440 and S240-5-20, both from Oregon State Univ.). The range of similarity using SRAP markers varied between 0.43 (between USVL032 and USVL059 derived from 'Green Valiant' and 'Packman', respectively), and 0.84 (between USVL012 and USVL089, both derived from 'Everest'). AFLP markers had a range of similarity between 0.57 (between USVL105 and USVL106, derived from 'Arcadia' and 'Major', respectively) and 0.87 (between S440 and S240-5-20, both from Oregon State Univ; between USVL047 and USVL073, both derived from 'Marathon'; and between USVL032 and USVL039, derived from 'Green Valiant' and 'High Sierra', respectively). Overall, AFLPs had the highest similarity values, and SRAPs had the lowest, while SSRs had the largest range in genetic similarities (Table 3). Differences in rates of polymorphism were expected since the SRAPs preferentially amplify expressed regions, AFLPs amplify both expressed and nonexpressed regions, and SSRs tend to amplify nonexpressed regions (Ferriol et al, 2004).

Dendrograms were generated based on each marker type and a combination of all three (Fig. 1A-D). While the topology of each tree is unique, there are evident similarities among them. First, the derivatives of 'Everest' (USVL012, 089, and 013) always grouped together with USVL070 (derivative of 'Futura') with all three marker sets. Second, the inbreds from Oregon State Univ. (S440 and S240-5-20) grouped together regardless of the marker system used as did USVL111 and USVL110, two lines selected for adaptation to hot summer conditions. In addition to the above commonalities, USVL047 and 048 (derived from 'Marathon'), USVL032 (derived from 'Green Valiant'), USVL039 (derived from 'High Sierra'), and USVL105 (derived from 'Arcadia') grouped together with all three markers. USVL066 (derived from 'Viking') and USVL073 (derived from 'Marathon') were also positioned within the latter group by AFLPs, and SRAPs, but SSRs clustered them differently. USVL106 and USVL109 were also found clustered together regardless of marker type, however, their positions with respect to the other lines varied, based on marker type. Discrepancies in formation of subgroups within major groups were observed as well as in the positioning of the remaining inbred lines. Inbred lines derived from the Cornell Breeding Program germplasm (USVL112 and USVL113) as well as USVL093 exemplify genotypes that always grouped differently depending on the marker type used.

COMPARISON BETWEEN MARKER SYSTEMS. Mantel test comparisons of dendrograms created using cophenetic and similarity matrices based on different sets of markers were all highly significant, indicating that there was a correlation between matrices. Despite the significance of the Mantel tests, the three marker systems showed slightly different groupings of the 24 genotypes. Correlation coefficients between cophenetic and similarity matrices were low to medium. Among the cophenetic matrices, the highest
Table 3. Mean, minimum, and maximum values of Dice similarity coefficient among 24 broccoli inbreds using different PCR-based markers.

\begin{tabular}{lcccc}
\hline & & \multicolumn{3}{c}{ Genetic similarity } \\
\cline { 3 - 5 } Marker set & Loci (no.) & Maximum & Minimum & Mean \\
\hline SSR & 55 & 0.91 & 0.32 & 0.64 \\
AFLP & 311 & 0.87 & 0.57 & 0.69 \\
SRAP & 255 & 0.84 & 0.43 & 0.62 \\
AFLP-SRAP-SSR & 621 & 0.87 & 0.54 & 0.66 \\
\hline
\end{tabular}

correlation was found between SSR and SRAP (0.58) followed by AFLP and SRAP (0.45) and AFLP and SSR (0.35). The correlation coefficients of the similarity matrices were somewhat different, with the highest coefficient between SSR and SRAP (0.5) followed by AFLP and SSR (0.35) and SRAP and AFLP (0.2) (Table 4). In general, those groupings with relatively high bootstrap confidence limits based on the WinBoot program tended to group the same regardless of the marker system, and those with lower confidence limits grouped differently. The confidence limits were much lower for SSRs than for any other type of marker, presumably because of the low number of bands generated in this evaluation.

The cophenetic correlation coefficient is used as a measure of goodness of fit for a cluster analysis. It indicates how well the clustering of genotypes accurately represents the estimates of genetic similarity obtained with each respective marker system (Pejic et al., 1998). Overall, the cophenetic correlation coefficients showed intermediate values that were not extremely different between the three marker systems. SRAPs had the highest correlation (0.78) followed by SSRs (0.77) and AFLPs (0.74). When all three sets of markers were combined into a single data set and analyzed together, the cophenetic correlation was 0.81 , which is generally considered to be a high value (Rohlf, 2002). This indicates that a combination of the three marker systems gives a better estimate of similarity than any single marker type alone.

A majority of inbred lines used in this study were derived from commercial hybrids and the pedigree information for these hybrids is considered a trade secret and not available. Thus, the true relationships among the inbreds at the pedigree level are not known. Lacking this information, at least one assumption can be made regarding the similarities among lines. Mainly, inbreds derived from the same hybrid are more similar than those derived from different hybrids. Despite differences in the dendrograms, all three marker systems reflected this assumption.

The low correlation between the groupings obtained with different marker systems probably reflects the differential sampling of the genome provided by each of the markers. SRAP markers preferentially target expressed regions of the genome and a previous study by Li and Quiros (2001) on B. olearacea showed that $\approx 45 \%$ of gel isolated bands matched known genes in the Genebank database. In contrast to SRAPs, AFLP markers obtained using EcoRI/MseI enzymes amplify both coding and noncoding regions of the genome, and can be unevenly distributed across the genetic map. Studies carried out with tomato (Lycopersicon pennelli L.) and sorghum [Sorghum bicolor (L.) Moench] have shown that these markers are mainly clustered in centromeric regions (Bonnema et al., 2002; Menz et al., 2002). Thus, it is probable that the centromeric regions are overrepresented with AFLP markers in $B$. oleracea as well, weighing these regions more heavily when grouping germplasm. Menz et al. (2004) suggest that Pst I/MseI AFLP genetic markers might be a better marker choice on crops where saturated genetic maps do not yet 


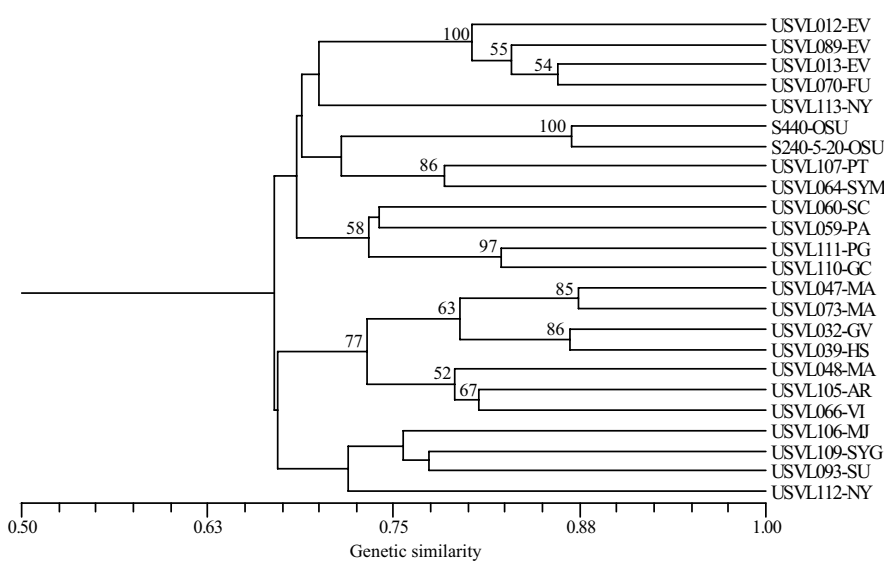

B) Based on SSR markers

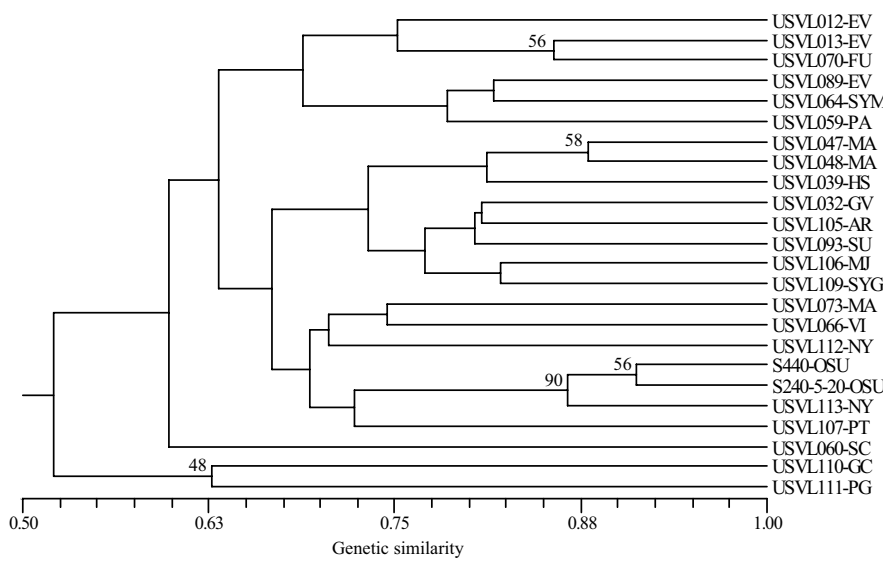

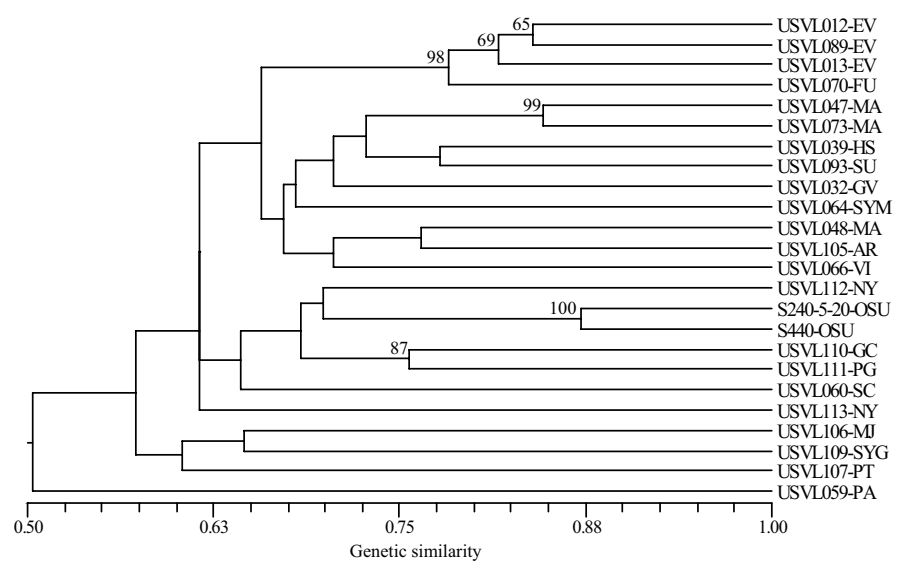

D) Based on combination of all three (AFLP, SSR, SRAP) markers

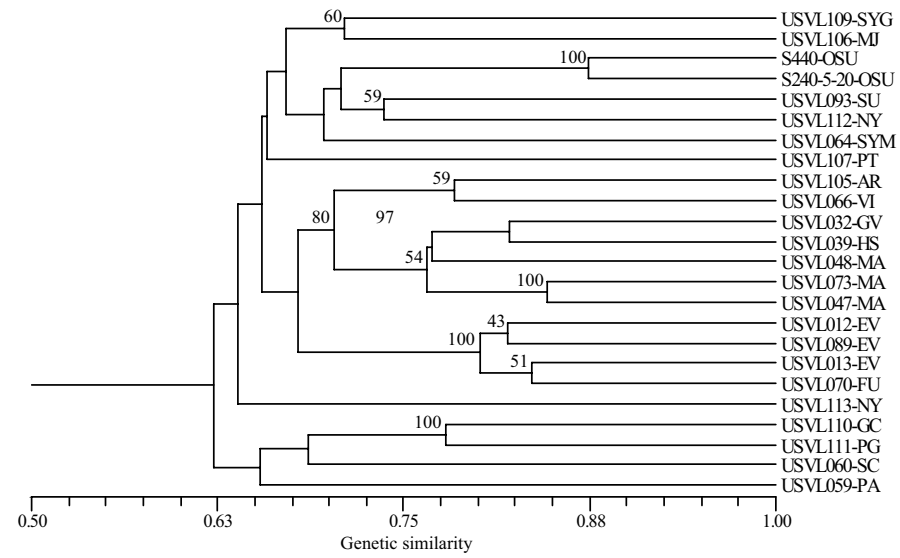

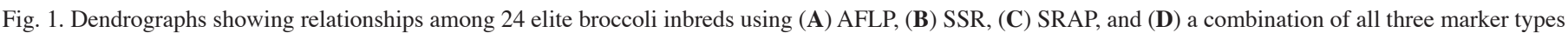

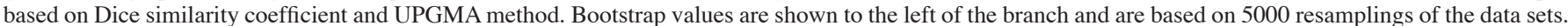

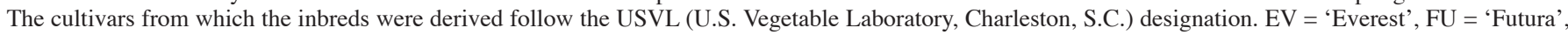

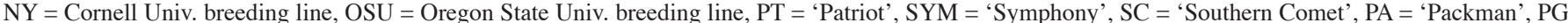

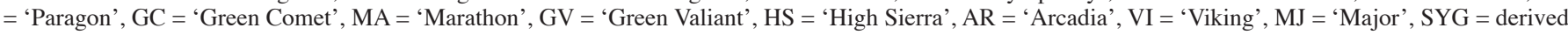
from a Syngenta line, and SU = 'Sultan'.

Table 4. Correlation between cophenetic matrices (above diagonal) and similarity matrices (below diagonal) obtained with different marker sets. Cophenetic correlation coefficients for the dendrograms are in bold on the diagonal.

\begin{tabular}{llll}
\hline & SSR & AFLP & SRAP \\
\hline SSR & $\mathbf{0 . 7 7}$ & 0.35 & 0.58 \\
AFLP & 0.35 & $\mathbf{0 . 7 4}$ & 0.45 \\
SRAP & 0.5 & 0.2 & $\mathbf{0 . 7 8}$ \\
\hline
\end{tabular}

exist since these markers amplify largely unmethylated gene-rich regions of the genome. All SSR primers were publicly available sequences which were chosen at random, and their distribution across the $B$. oleracea genome is not known. It is probable that the modest number of SSR markers were not evenly distributed and possible that entire chromosomes were not represented with the SSR data. This, along with the relatively low number of markers obtained with this system could explain the differences between this and the other marker types. It was suggested by AlmanzaPinzon et al. (2003) that since SSRs evolve more quickly than other marker types, they may provide a more accurate measurement of similarity in highly related lines, and decreased accuracy as the lines become less related.
Each of these marker systems was able to discriminate between the 24 elite inbreds for fingerprinting purposes, however, when determining relatedness, the confidence levels could be increased by combining marker systems and/or using more of each type of primers. Each of these techniques has some obvious advantages over the older marker systems. The high multiplex ratio of AFLPs along with the reported repeatability make them very efficient at generating large numbers of markers. The possibly uneven distribution of $E c o$ RI/MseI could lead to some difficulties when trying to map under-represented regions, but switching to AFLPs generated from enzymes such as $P$ stI $/ M s e I$, which are more evenly distributed in some species, could overcome this problem. SRAP markers are reported to be tightly linked to coding regions and a linkage map in $B$. oleracea showed that they were evenly distributed across the nine major linkage groups ( $\mathrm{Li}$ and Quiros, 2001). Moreover, it has been reported that $20 \%$ of the SRAP markers are co-dominant whereas almost all AFLP primers are dominant (Li and Quiros, 2001). Although SSRs have the advantage of being co-dominant, they were the least efficient and most time-consuming of the marker systems analyzed. AFLPs and SRAPs yielded higher confidence limits and required fewer primers to discriminate among the inbreds making them better choices to replace older techniques. However, the results of this 
study demonstrate that using more than one marker system increases the amount of the genome that is sampled and probably gives a more accurate estimate of genetic similarity.

\section{Literature Cited:}

Almanza-Pinzon, M.I., M. Khairallah, P.N. Fox, and M.L. Warburton. 2003. Comparison of molecular markers and coefficients of parentage for the analysis of genetic diversity among spring bread wheat accessions. Euphytica 130:77-86.

Becker, J., P. Vos, M. Kuiper, F. Salamini, and M. Heun. 1995. Combined mapping of AFLP and RFLP markers in barley. Mol. Gen. Genet. 249:65-73.

Bonnema, G.,P. van der Berg, and P. Lindhout. 2002.AFLPs mark different genomic regions compared with RFLPs: A case study in tomato. Genome 45:217-221.

Bowers, J.E., G.S. Dangl, R. Vignani, and C.P. Meredity. 1996. Isolation and characterization of new polymorphic simple sequence repeat loci in grape (Vitis vinifera L.). Genome 39:628-633.

Breyne, P., D. Rombaut, A. van Gysel, M. Van Montagu, and T. Gerats. 1999. AFLP analysis of genetic diversity within and between Arabidopsis thaliana ecotypes. Mol. Gen. Genet. 261:627-634.

Camargo, L.E.A., L. Savides, G. Jung, J. Nienhuis, and T.C. Osborne. 1997. Location of the self-incompatibility locus in an RFLP and RAPD map of Brassica oleracea. J. Heredity 88:57-60.

Divaret, I., E. Margale, and G. Thomas. 1999. RAPD markers on seed bulks efficiently assess the genetic diversity of Brassica oleracea L. collection. Theor. Appl. Genet. 98:1029-1035.

dos Santos, J.B., J. Neinhuis, P. Skroch, J. Tivang, and M.K. Slocum. 1994. Comparison of RAPD and RFLP genetic markers in determining genetic similarity among Brassica oleracea L. genotypes. Theor. Appl. Genet. 87:909-915.

Farnham, M.W. 1996. Genetic variation among and within United States collard cultivars and landraces as determined by randomly amplified polymorphic DNA markers. J. Amer. Soc. Hort. Sci. 121:374-379.

Farnham, M.W. 1998. Doubled haploid broccoli production using anther culture: Effect of anther source and seed set characteristics of derived lines. J. Amer. Soc. Hort. Sci. 123:73-77.

Ferriol, M., B. Pico, and F. Nuez. 2004. Morphological and molecular diversity of a collection of Cucurbita maxima landraces. J. Amer. Soc. Hort. Sci. 129:60-69.

Giovannelli, J.L., M.W. Farnham, M. Wang, and A.E. Strand. 2002. Development of sequence characterized amplified region markers linked to downy mildew resistance in broccoli. J. Amer. Soc. Hort. Sci. 127:597-601.

Haanstra, J., C. Wye, H. Verbakel, F. Meijer-Dekens, P. van den Berg, P. Odinot, A.W. van Heusden, S. Tanksley, P. Lindhout, and J. Pelemen. 1999. An integrated high-density RFLP-AFLP map of tomato based on two Lycopersicum esculentum $\mathrm{x}$ L. penellii $\mathrm{F}_{2}$ populations. Theor. Appl. Genet. 99:254-271.

Holme, I.B., A.M. Torp, L.N. Hansen, and S.B. Andersen. 2004. Quantitative trait loci affecting plant regeneration from protoplasts of Brassica oleracea. Theor. Appl. Genet. 108:1513-1520.

$\mathrm{Hu}$, J. and C.F. Quiros. 1991. Identification of broccoli and cauliflower cultivars with RAPD markers. Plant Cell Rpt. 10:505-511.

Kaminski, P., M. Staniaszek, and E.U. Kozik. 2003. Evaluation of genetic diversity and uniformity of head cabbage DH lines by the use of RAPD markers. J. Appl. Genet. 44:157-163.

Klein, P.E., R.R. Klein, S.W. Cartinhour, P.E. Ulanch, J. Dong, J.A. Obert, D.T. Morishige, S.D. Schlueter, K.L. Childs, M. Ale, and J.E. Mullet. 2000. A highthroughputAFLP-based method for constructing integrated genetic and physical maps: Progress toward a sorghum genome map. Genome Res. 10:789-807.

Kresovich, S., J.G. K. Williams, J.R. McFerson, E.J. Routman, and B.A. Schaal. 1992. Characterization of genetic identities and relationships of Brassica oleracea L. via a random amplified polymorphic DNA assay. Theor. Appl. Genet. 85:190-196.

Kresovich, S., A.K. Szewc-McFadden, S.M. Bliek, and J.R. McFerson. 1995. Abundance and characterization of simple-sequence repeats (SSRs) isolated from a size-fractioned genomic library of Brassica napus L. (rapeseed). Theor. Appl. Genet. 91:206-211.

Lanner-Herrera, C., M. Gustafsson, A.S. Falt, and T. Bryngelsson. 1996. Diversity in natural populations of wild Brassica oleracea as estimated by isozyme and RAPD analysis. Genet. Resources Crop Evol. 43:13-23.
Lee, D., J.C. Reeves, and R.J. Cooke. 1996. DNA profiling and plant variety registration: 1. The use of random amplified DNA polymorphisms to discriminate between varieties of oilseed rape. Electrophoresis 17:261-265.

Li, G. and C.F. Quiros. 2001. Sequence-related amplified polymorphism (SRAP), a new marker system based on a simple PCR reaction: Its application to mapping and gene tagging in Brassica. Theor. Appl. Genet. 103:455-461.

Lowe, A. J., A.E. Jones, A.F. Raybould, M.Trick, C.L. Moule, and K.J. Edwards. 2002. Transferability and genome specificity of a new set of microsatellite primers among Brassica species of the U triangle. Mol. Ecol. Notes 2:7-11.

Mantel, N. 1967. The detection of disease clustering and generalized regression approach. Cancer Res. 27:209-220.

Menz, M.A., R.R. Klein, J.E. Mullet, J.A. Obert, N.C. Unruh, and P.E. Klein. 2002. A high density genetic map of Sorghum bicolor (L.) Moench based on 2926 AFLP, RFLP, and SSR markers. Plant. Mol. Biol. 48:483-499.

Menz, M.A., R.R. Klein, N.C. Unruh, W.L. Rooney, P.E. Klein, and J.E. Mullet. 2004. Genetic diversity of public inbreds of sorghum determined by mapped AFLP and SSR markers. Crop Sci. 44:1236-1244.

Neinhuis, J., M.K Slocum, D.A. De Vos, and R. Mureen. 1993. Genetic similarity among Brassica oleracea L. genotypes as measured by restriction fragment length polymorphisms. J. Amer. Soc. Hort. Sci. 118:298-303.

Nei, M. and W. Li. 1979. Mathematical model for studying genetic variation in terms of restriction endonucleases. Proc. Natl. Acad. Sci. USA 79:5269-5273.

Pejic, I., P. Ajmone-Marsan, M. Morgante, V. Kozumplik, P. Castiglioni, G. Taramino, and M. Motto. 1998. Comparative analysis of genetic similarity among maize inbred lines detected by RFLPs, RAPDs, SSRs, and AFLPs. Theor. Appl. Genet. 97:1248-1255.

Phippen, W.B., S. Kresovich, F.G. Candelas, and J.R. McFerson. 1997. Molecular characterization can quantify and partition variation among genebank holdings: A case study with phenotypically similar accessions of Brassica oleracea var. capitata L. (cabbage) 'Golden Acre'. Theor. Appl. Genet. 94:227-234.

Plieske, J. and D. Struss. 2001. Microsatellite markers for genome analysis in Brassica. I. development in Brassica napus and abundance in Brassicaceae species. Theor. Appl. Genet. 102:689-694.

Rohlf, F.J. 2002. NTSYSPC. Numerical taxonomy system. Exter Software, New York.

Saal, B., J. Plieske, J. Hu, C.F. Quiros, and D. Struss. 2001. Microsatellite markers for genome analysis in Brassica. II. Assignment of rapeseed microsatellites to the $\mathrm{A}$ and $\mathrm{C}$ genomes and genetic mapping in Brassica oleracea $\mathrm{L}$. Theor Appl. Genet. 102:695-699.

Sebastian, R.L., E.C. Howell, G.J. King, D.F. Marshall, and M.J. Kearsey. 2000. An integrated AFLP and RFLP Brassica oleracea linkage map from two morphologically distinct doubled-haploid mapping populations. Theor. Appl. Genet. 100:75-81.

Shi, J., R. Ward, and D. Wang. 2001. Application of a high throughput, low cost, non-denaturing polyacrylamide gel system for wheat microsatellite mapping. 2001 Natl. Fusarium Head Blight Forum. p. 25-30.

Sneath, P.H.A. and R.R. Sokal. 1973. Numerical taxonomy. W.H. Freeman, San Francisco.

Sobotka, R., L. Dolanska, V. Curn, and J. Ovensa. 2004. Fluorescence-based AFLPs occur as the most suitable marker system for oilseed rape cultivar identification. J. Appl. Genet. 45:161-173.

Song, K.M., T.C. Osborn, and P.H. Williams. 1988. Brassica taxonomy based on nuclear restriction fragment length polymorphisms (RFLPs) I. Genome evolution of diploid and amphidiploid species. Theor. Appl. Genet. 75:784-794.

Suwabe, K., H. Iketani, T. Nunome, T. Kage, and M. Hirai. 2002. Isolation and characterization of microsatellites in Brassica rapa L. Theor. Appl. Genet. 104:1092-1098.

Szewc-McFadden, A.K., S. Kresovich, S.M Bliek, S.E. Mitchell, and J.R. McFerson. 1996. Identification of polymorphic, conserved simple sequence repeats (SSRs) in cultivated Brassica species. Theor. Appl. Genet. 93:534-538.

Vos, P., R. Hogers, M. Bleeker, M. Reijans, T. van de Lee, M. Hornes, A. Frijters, J. Pot, J. Peleman, M. Kuiper, and M. Zabeau. 1995. AFLP: A new technique for DNA fingerprinting. Nucleic Acids Res. 23:4407-4414.

Williams, J.G.K., A.R. Kubelik, K.J. Livak, J.A. Rafalski, and S.V. Tingey. 1990. DNA polymorphisms amplified by arbitrary primers are useful as genetic markers. Nucleic Acids Res. 18:6531-6535.

Yap, I.V. and R.J. Nelson. 1996. WinBoot: A program for performing bootstrap analysis of binary data to determine the confidence limits of UPGMA-based dendrograms. Intl. Rice Research Inst., Manila, Philippines. 